\title{
Palmilhas de reprogramação postural na diminuição da pressão arterial e do desalinhamento postural em indivíduos hipertensos: ensaio clínico randomizado
}

\section{Postural reprogramming insoles for blood pressure fall and posture misalignment in hypertensive individuals: randomized clinical trial}

\author{
Ana Lucia Barbosa Goes ${ }^{1}$ (i) \\ Alana Santana Menezes Barbosa ${ }^{2}$ (1) \\ Beatriz Guedes Ventura Araújo3 3 \\ Camila Barbosa de Castro 4 \\ Géssica Marília de Oliveira Gazar Barbalho ${ }^{5}$ (1) \\ Vinícius Cardoso Lago 6 (1) \\ Luis Agnaldo Pereira de Souza7 \\ Ana Marice Teixeira Ladeia ${ }^{8}$
}

\begin{abstract}
${ }^{1}$ Autora para correspondência. Escola Bahiana de Medicina e Saúde Pública (Salvador), Universidade Federal da Bahia (Salvador). Bahia, Brasil. albgoes@bahiana.edu.br ${ }^{2-8}$ Escola Bahiana de Medicina e Saúde Pública (Salvador). Bahia, Brasil. alanabarbosa15.1@bahiana.edu.br, beatrizgaraujo15.1@bahiana.edu.br, camilacastro15.1@bahiana.edu.br, gessicabarbalho15.1@bahiana.edu.br, vc.lago@hotmail.com, lagnaldosouza@bahiana.edu.br,
\end{abstract} anamarice@bahiana.edu.br

RESUMO | OBJETIVO: Testar a hipótese que Palmilhas de Reprogramação Postural (PRP) melhoram a postura e podem modificar média e picos de pressão arterial (PA) em indivíduos hipertensos. DESENHO DE ESTUDO: ECR, registrado no Clinical Trials (NCT02401516), com 24 indivíduos hipertensos. CONFIGURAÇÃO: Todos foram submetidos à Monitorização Ambulatorial da Pressão Arterial (MAPA) e avaliação da postura (SAPO) no início e após seis semanas. INTERVENÇÃO: grupo intervenção (GI) utilizou PRP e grupo controle (GC) SHAM. DESFECHO PRIMÁRIO: Médias da PA. DESFECHOS SECUNDÁRIOS: Picos de PA, durante vigília e sono e ângulo posturais. A melhora da postura ocorreu quando valores angulares diminuíram ou chegaram a zero. Para comparar a PA, foram utilizados os testes t de Student, pareado e não-pareado, com nível de significância de 5\%. O tamanho do efeito (TDE) foi avaliado com D de Cohen. Para postura, Wilcoxon e Mann-Whitney. Variáveis categóricas, através do teste do Qui-quadrado. RESULTADOS: As variáveis de linha de base não diferiram entre grupos. Foram obtidos os seguintes deltas: pico da PAS em vigília $(+9,3 \mathrm{mmHgVs}-7,5 \mathrm{mmHg})(p<0,05$, grande TDE); pico da PAS no sono $(+2,3 \mathrm{mmHgVs}-6,8 \mathrm{mmHg})(p<0,05$, TDE moderado); e pico da PAD durante vigília $(+3,2 \mathrm{mmHgVs}-4,7 \mathrm{mmHg})(p<0,05$, grande TDE), GC e GI respectivamente. Para os ângulos posturais, 33\% da PAS foram explicados pelo deslocamento anterior do corpo. Para a PAD, 46\% e 55\%, pelos ângulos de Joelho e Tornozelo, respectivamente. CONCLUSÕES: A PRP reduziu picos de PAS e PAD durante vigília, sem efeito na média da PA. Embora PRP não tenha mostrado melhora na postura geral, o deslocamento anterior do corpo e os ângulos de joelho e tornozelo explicaram 33-55\% da PA mais alta.

PALAVRAS-CHAVE: Hipertensão arterial. Palmilhas. Pressão arterial. Postura.
ABSTRACT | OBJECTIVE: To test the hypothesis that Postural Reprogramming Insoles (PRI) improves posture and the effect of PRI on average and peaks of blood pressure (BP) in hypertensive individuals. DESIGN: RCT, registered at the Clinical Trials (NCT02401516), with 24 hypertensive individuals. SETTING: All patients underwent Ambulatory Blood Pressure Monitoring (ABPM) and posture assessment (PAS/SAPO software) at the beginning and the end of six weeks. INTERVENTION: intervention group (IG) used PRI and control group (CG) SHAM. MAIN OUTCOME MEASURES: BP averages. SECONDARY OUTCOMES: BP peaks, during awake and asleep periods. Improvement of posture was determined when the angle values assessed decreased or reached zero (perfect alignment). To compare BP, paired, and unpaired Student's t-tests were used, significance level of 5\%. Effect size (ES) was assessed with Cohen's D. For posture assessment, Wilcoxon and Mann-Whitney were applied to analysis. Categorical variables, through Chi-square test. RESULTS: Baseline variables did not differ between groups. The following deltas were obtained-SBP peak in awake period $(+9.3 \mathrm{mmHgVs}-7.5 \mathrm{mmHg})(p<0.05$, great ES); SBP peak during sleeping period $(+2.3 \mathrm{mmHgVs}-6.8 \mathrm{mmHg})(p<0.05$, moderate ES); and DBP peak during awake period $(+3.2 \mathrm{mmHgVs}-4.7 \mathrm{mmHg})(p<0.05$, great $E S)$, in control and intervention groups, respectively. For postural angles, 33\% of SBP were explained by anterior body shift. For DBP, $46 \%$ and 55\% were explained by Knee and Ankle Angles, respectively. CONCLUSIONS: PRI reduced SBP and DBP peaks during awake period, with no effect on BP average. Even though PRI has not shown any improvement in overall posture, anterior body displacement and knee and ankle angles would solely explain $33-55 \%$ of the highest BP.

KEYWORDS: Hypertension. Insoles. Blood pressure. Posture. 


\section{Introdução}

Hipertensão Arterial Sistêmica (HAS) é um dos principais fatores de risco modificáveis para doenças do aparelho circulatório ${ }^{12}$. Estima-se que cerca de $17 \mathrm{mi}$ Ihões de mortes/ano em todo o mundo sejam causadas por doenças do aparelho circulatório, e dessas, 55,3\% relacionadas a elevação da pressão arterial $(\mathrm{PA})^{3}$. Excessiva ativação do sistema nervoso simpátiCo (SNS) parece ter papel importante na manutenção da HAS ${ }^{4-6}$, sendo considerado como o sistema final de integração na regulação da função cardiovascular ${ }^{7}$. A maioria dos nervos vasculares do SNS causam vasoconstricção, seu transmissor principal é noradrenalina, sofre interferência dos sistemas musculoesqueléticos, vestibular, tegumentar ${ }^{7}$, respiratório ${ }^{8}$, renal, endócrino 9 e somatosensorial ${ }^{10}$.

Postura pode ser definida como a forma que o corpo adquire em determinado momento para se contrapor a ação da força gravitacional aplicada a cada segmento corporal, mantendo posicionamento estável desses segmentos ${ }^{11}$. A posturologia trata alterações posturais e tem como base o uso de palmilhas para devolver a postura adequada ao indivíduo. A palmilha de reprogramação postural (PRP) é composta por dispositivo em relevo, no centro do médio-pé, constituído por dois processos polarizantes cruzados, formado por macromoléculas ferromagnéticas, orientadas à quente por laminagem e que gera corrente elétrica e provoca vibração ${ }^{12}$. Apesar de não saber exatamente como este dispositivo realmente funciona, parece que essa corrente estimula, via sistema autônomo, o Sistema Tônico Postural (STP), de forma permanente com o pé apoiado ou não $\frac{13}{13}$, promovendo a adequação da postura ${ }^{14-18}$.

Em associação, um estudo desenvolvido por este grupo encontrou um possível padrão de desalinhamento postural entre indivíduos hipertensos: deslocamento anterior do tronco, deslocamento posterior do corpo, flexão do quadril, joelho e tornozelo. As alterações desses ângulos causaram maiores cargas pressóricas na MAPA, para carga total, durante os períodos de vigília ou de sono, e menores variações pressóricas entre o período vigília/sono, tanto para a pressão arterial sistólica quanto diastólica. Estes achados sugerem associação entre desalinhamento postural e alteração da $\mathrm{PA}^{19}$.
As vias utilizadas pelo sistema nervoso autônomo (SNA) para controle da $\mathrm{PA}^{9}$ parecem ser semelhantes às vias utilizadas para o controle da postura, às quais são utilizadas também pela PRP para adequação postural13. Devido a esta semelhança nas áreas de ativação, acredita-se que a PRP possa apresentar algum efeito na regulação da PA. Assim, torna-se importante analisar a possível influência da PRP no controle da PA em indivíduos hipertensos.

Portanto, esse estudo teve como objetivo avaliar o efeito da PRP sobre a queda da PA e o desalinhamento postural em indivíduos hipertensos.

\section{Materiais e métodos}

\section{Desenho de estudo}

Trata-se de ensaio clínico controlado e randomizado, triplo cego, exploratório, realizado com indivíduos hipertensos acompanhados em ambulatório vinculado à instituição de ensino e em centro de atenção primária a saúde, no período de 20 meses.

\section{Participantes}

Os critérios de inclusão para o estudo foram: diagnóstico prévio de hipertensão ( $S B P \geq 140 \mathrm{mmHg}$ e/ou $\mathrm{DBP} \geq 90 \mathrm{mmHg}$ ), durante pelo menos dois meses, ambos os sexos, idade entre 30-60 anos, índice de massa corporal (IMC) até $34,9 \mathrm{~kg} / \mathrm{m} 2$, e estabilidade clínica. Todos os participantes foram instruídos a manter padrão dietético e medicamentos prescritos.

Foram excluídos do estudo indivíduos com história de doenças cerebrovasculares e eventos cardiovasculares prévios (infarto do miocárdio, insuficiência cardíaca, angina instável), doença renal ou arterial periférica diagnosticadas, doenças neurológicas, mentais, gravidez, Diabetes Melito, participantes que usavam medicação para controle glicêmico e os que não realizaram MAPA pós-intervenção.

Este estudo foi aprovado pelo Comité de Ética da Escola Bahiana de Medicina e Saúde Pública (EBMSP) sob CAAE 16952113.5.0000.5544. A concepção do projeto baseou-se no checklist CONSORT 20 . A identificação deste estudo no site ClinicalTrials.gov é NCT02401516. 


\section{Intervenção e Randomização}

Os participantes foram alocados em dois grupos por meio de sorteio. Ambos os grupos utilizaram palmilhas, e no grupo intervenção $(\mathrm{Gl})$, o PRP continha o ressonador que emite a corrente eletromagnética, enquanto que a palmilha do grupo controle (GC) continha um dispositivo com dimensões e tamanho semelhantes ao ressonador, porém feito de cortiça. Os participantes foram instruídos a utilizar a palmilha durante pelo menos 12 horas por dia, por 6 semanas.

Após assinatura do termo de consentimento livre e esclarecido (TCLE), os participantes compareceram ao Laboratório de Pesquisa Cardiovascular-EBMSP, para colocação da MAPA, de acordo com a V Diretriz Brasileira para uso da MAPA 21 . A MAPA foi realizada um dia antes da colocação da PRP para definir valores de PA, considerados como basais e nova avaliação foi realizada seis semanas após o uso da palmilha. Os participantes foram orientados para completar um diário de atividades com dados sobre sintomas e outras situações que poderiam modificar a PA durante o período de intervenção²1.

\section{Avaliação da Postura}

Hemiesferas de isopor de $25 \mathrm{~mm}$ de diâmetro foram coladas nos principais acidentes ósseos com fita dupla face (marca 3M), de acordo com protocolo SAPO. Os pés foram posicionados em abdução de $30^{\circ}$ para alinhamento e padronização das imagens. Sujeitos trajados de short e top (mulheres) e short (homens) foram posicionados sobre papel metro, com área de $1 \mathrm{~m} 2$, próximos ao fio de prumo, preso ao teto e com marcação de $10 \mathrm{~cm}$ de comprimento para fins de calibração da imagem no software. As Imagens foram capturadas pela câmera fotográfica digital da marca Sony Cybershot (modelo DSC-W570, 16,1 megapixels), apoiada em tripé, disposto a três metros de distância do sujeito e na metade da sua altura.

O programa gera relatório nas duas vistas laterais e foi sorteada a Vista Lateral Direita. Os ângulos foram descritos como: 1) Alinhamento Vertical do Tronco (AVT), formado entre acrômio, trocanter maior e linha vertical; 2) Alinhamento Vertical do Corpo (AVCo), formado entre acrômio, maléolo lateral e linha vertical; 3) Ângulo do Quadril (AQ), formado entre acrômio, trocanter maior e maléolo lateral; 4) Ângulo do Joelho (AJ), formado entre trocanter maior, linha articular lateral do joelho e maléolo lateral e 5) Ângulo do tornozelo (AT), formado pela linha articular lateral do joelho e as linhas lateral e horizontal do maléolo lateral. (Figura 1).

Figura 1. Ângulos utilizados no estudo na Vista Lateral Direita
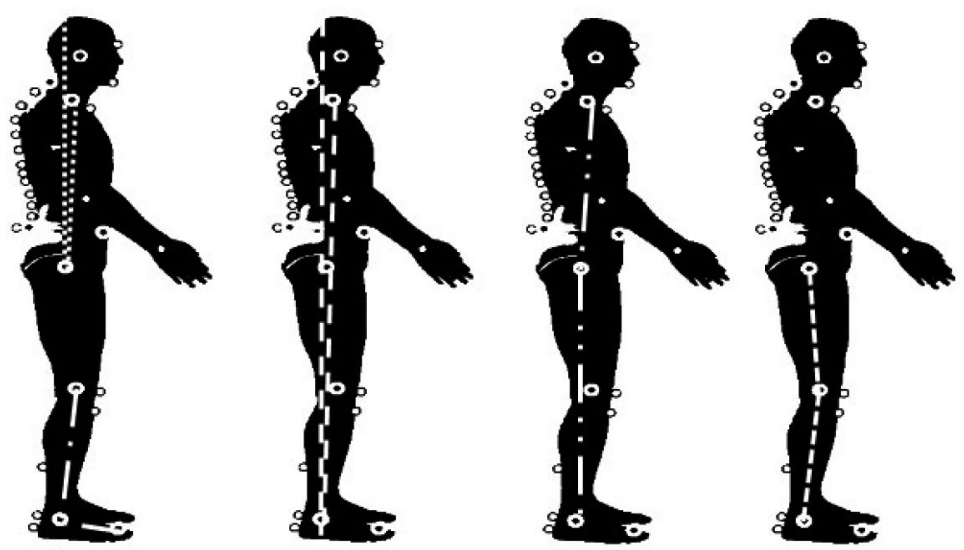


\section{Desfecho Primário}

Médias das Pressões Arterial Sistólica e Diastólica.

\section{Desfecho Secundário}

Picos pressóricos da PA Sistólica e Diastólica (PAS e $P A D)$, durante vigília e sono e ângulos posturais.

Após validação do exame, questionário contendo informações sociodemográficas, de hábitos de vida e saúde foi preenchido. Peso e estatura foram avaliados na balança manual da marca Welmy ${ }^{\circledR}$ (Santa Bárbara D'Oeste, SP). A PA de consultório foi medida conforme a VII Diretriz Brasileira de Hipertensão², por meio de aparelho digital automático de braço, modelo HEM-742, da marca Omron (Omron Healthcare Inc., Lake Forest, IL, USA). Foram realizadas três medidas com um minuto de intervalo entre elas e calculada média aritmética. Todas as avaliações foram realizadas por pesquisador cego e treinado para este fim.

Para avaliação dos efeitos imediatos, as aferições da PAS e PAD, em consultório, foram realizadas um, cinco e dez minutos após a colocação das palmilhas para ambos os grupos e os efeitos tardios foram avaliados com aferições dos picos e médias da PA, realizadas após seis semanas de uso contínuo das palmilhas, com nova MAPA.

Para postura, os seguintes ângulos foram avaliados: Alinhamento Vertical do Tronco (AVT) e Alinhamento Vertical do Corpo (AVCo), categorizados em deslocamento posterior (quando valores angulares negativos) e anterior (valores positivos); Ângulo do Quadril (AQ), categorizado em aumentado (quando valores angulares negativos, caracterizando extensão), e diminuído (valores positivos, caracterizando flexão); Ângulo do Joelho (AJ), categorizado em aumentado (quando valores angulares negativos, caracterizando hiperextensão ou geno recurvato), e diminuído (valores positivos, caracterizando semiflexão). Ângulo do tornozelo (AT), categorizado em aumentado (valores angulares acima de $90^{\circ}$, caracterizando plantiflexão) e diminuído (valores abaixo, caracterizando dorsiflexão).

A melhora foi determinada quando o valor do ângulo diminuiu ou atingiu zero (alinhamento perfeito). Foi considerado pior quando os valores aumentaram ou foram invertidos (mudando entre positivo e negativo).
A análise de intenção de tratar não pôde ser realizada, porque o protocolo de estudo considerou não realizar MAPA após intervenção como critério de exclusão, uma vez que o estudo teve um curto período de tempo para realizar o exame. Tentou-se agendar o exame em dois momentos num intervalo de 10 dias. O participante que não compareceu, foi excluído do estudo. No entanto, mesmo após a aplicação deste protocolo, a aleatorização não foi comprometida.

\section{Amostra}

Considerando a metanálise desenvolvida por Lin²2, em que o controle dietético e atividade física realizados no período de 12 meses provocaram diminuição da PA em torno de $2,29 \mathrm{mmHg}$, optou-se por considerar desvio padrão da média da PA entre os grupos de $3 \mathrm{mmHg}$, para detectar diferença entre as médias da PA de $3 \mathrm{mmHg}$, nível de significância de $5 \%$, poder do teste de $80 \%$, em hipótese bicaudal, totalizando 32 sujeitos, sendo 16 em cada grupo. Foi utilizada a calculadora WinPepi e os dados foram organizados e analisados no programa SPSS 14.0 para Windows.

\section{Cegamento}

Todos os exames de MAPA foram realizados por um cardiologista com experiência no uso e análise do método e que foi cego a alocação dos participantes. As avaliações da BP ambulatorial foram realizadas por um investigador cego e treinado para este fim. Os participantes não sabiam em que grupo foram alocados. Apenas uma pessoa da equipe tinha conhecimento sobre a distribuição de PRP e essa pessoa não participou na captação dos dados.

\section{Estatística}

Análise descritiva e inferencial. Variáveis sociodemográficas: Idade (anos), cor da pele (Preta/ Parda/ Branca/ Amarela/ Indígena), escolaridade (até 4 anos de estudo, de 5-8 anos, de 9-11 anos e 12 ou mais anos), estado civil (Casado-União Estável/ Solteiro/ Viúvo/ Separado-Divorciado); antropométricas e de hábitos de vida e saúde: IMC (Kg/m2), tabagismo (nunca fumou/ fumante/ ex-fumante), consumo de bebida alcoólica (dicotômica), uso regular de medicamento (dicotômica), tipo de medicamento (categórica); Posturais: ângulos da postura e clínicas: Picos da PA durante Vigília e Sono $(\mathrm{mmHg})$, Média da PA ( $\mathrm{mmHg}$ ), e medidas de consultório da PAS, PAD e FC. 
Foi utilizada média e desvio-padrão para análise descritiva das variáveis quantitativas. Para as variáveis categóricas, número absoluto e proporção. Para identificar efeitos imediatos da palmilha, foram comparadas diferenças nas médias da PAS e PAD nos diferentes tempos estimados (repouso, um, cinco e 10 minutos) por meio do teste ANOVA para medidas repetidas e pós-teste de Bonferroni. Para identificar diferenças nas médias e picos de PAS e PAD entre GI e GC, foi utilizado o teste estatístico t de Student não-pareado. Para as variáveis intragrupo foi utilizado teste t de Student pareado. Para identificar efeitos da PRP na postura, intragrupo, utilizou-se teste de Wilcoxon e Qui-quadrado, na análise intergrupo. Para associação entre ângulos de postura e variáveis pressóricas, utilizou-se teste de Correlação de Pearson. Todos os testes foram considerados com nível de significância de 5\%. Nas comparações intergrupos calculou-se Tamanho Do Efeito (TDE) por meio do teste $D$ de Cohen e considerou-se valores de até 0,2 como pequeno efeito, entre $0,21-0,5$, efeito moderado e valores acima de 0,8 grande efeito $\underline{\underline{23}}$. O teste $\mathrm{D}$ de Cohen foi calculado por meio da calculadora RStats Effect Size
Calculator for t-Tests ${ }^{24}$. Os dados foram organizados no programa SPSS 14.0 para Windows.

\section{Resultados}

Durante a avaliação de elegibilidade, 200 possíveis participantes tinham diagnóstico de HAS. Destes, 150 foram excluídos: idade acima de 60 anos (90) e apresentando co-morbidades como diabetes mellitus, síndrome metabólica e lesão de órgãos-alvo (60). Dos 50 avaliados, 20 foram excluídos: não se enquadraram nos critérios de elegibilidade (10) e recusaram-se a participar da pesquisa (10). Dos 30 que concordaram em participar do estudo, 15 participantes foram alocados em cada grupo e todos receberam a intervenção proposta. Houve perda de seguimento de 3 (20\%) participantes em cada grupo. No grupo intervenção, eles não compareceram para avaliação da MAPA após seis semanas e, no controle, dois participantes não compareceram para última avaliação e um não se adaptou à palmilha (Figura 2).

Figura 2. Elegibilidade segundo fluxograma CONSORT

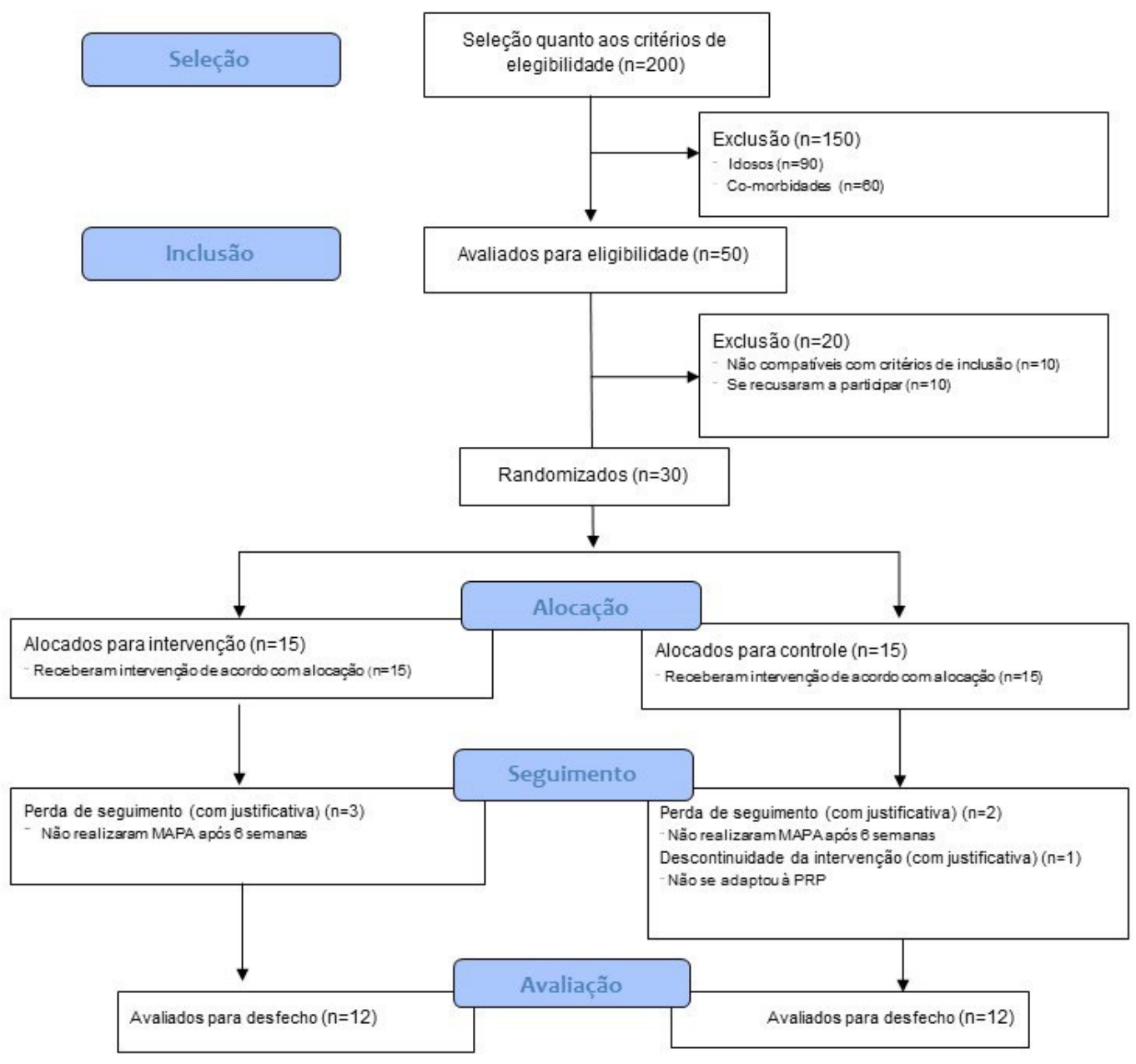


Foram incluídos 24 participantes, com média de idade de 49 $\pm 6,6$ anos, IMC de $30 \pm 4,2 \mathrm{Kg} / \mathrm{m} 2$. Ser do sexo feminino $(83,3 \%)$, casada $(54,2 \%)$, com 12 ou mais anos de estudo $(66,7 \%)$ e cor da pele preta ou parda (45,8\% para cada) foram as características mais frequentes. A maioria nunca fumou (70,8\%), não consumia bebida alcóolica (58,3\%), estava em uso regular de medicamento anti-hipertensivo (83,3\%). O medicamento mais frequente foi o bloqueador de receptores de angiotensina II (58,3\%) e a hipertensão estava controlada (70,8\%) (Tabela 1).

Tabela 1. Distribuição dos participantes de acordo com as características sociodemográficas e de estilo de vida. 2020. ( $n=24$ )

\begin{tabular}{|c|c|c|c|c|}
\hline & $\begin{array}{l}\text { Controle } \\
(n=12)\end{array}$ & $\begin{array}{l}\text { Intervenção } \\
(n=12)\end{array}$ & $\begin{array}{l}\text { Total } \\
(n=24)\end{array}$ & p-valor \\
\hline Variáveis & $\mathrm{X} \pm \mathrm{SD}$ & $\mathrm{X} \pm \mathrm{SD}$ & $\mathrm{X} \pm \mathrm{SD}$ & \\
\hline Idade (anos) & $47,8 \pm 7,5$ & $50,3 \pm 5,6$ & $49 \pm 6,6$ & $0,4^{\ddagger}$ \\
\hline $\mathrm{IMC}\left(\mathrm{Kg} / \mathrm{m}^{2}\right)$ & $30,4 \pm 3,4$ & $29,5 \pm 4,9$ & $30 \pm 4,2$ & $0,6^{\$}$ \\
\hline Sexo & $n(\%)$ & $n(\%)$ & & $1,0^{\$}$ \\
\hline Feminino & $10(83)$ & $10(83)$ & $20(83)$ & \\
\hline Estado Civil & & & & $0,34^{* *}$ \\
\hline Casada/Relacionamento Estável & $5(41,7)$ & $8(66,7)$ & $13(54)$ & \\
\hline Solteira & $4(33,3)$ & $2(16,7)$ & $6(25)$ & \\
\hline Separada/Divorciada & $2(16,7)$ & $1(8,3)$ & $3(12,5)$ & \\
\hline Viúva & $1(8,3)$ & $1(8,3)$ & $2(8,5)$ & \\
\hline Escolaridade (anos) & & & & $0,33^{* *}$ \\
\hline Até 4 & $1(8,3)$ & --- & $1(4,2)$ & \\
\hline $5-8$ & ---- & $2(16,7)$ & $2(8,4)$ & \\
\hline $9-11$ & $1(8,3)$ & $4(33,3)$ & $5(20,8)$ & \\
\hline 12 ou mais & $10(83,3)$ & $6(50)$ & $16(66,6)$ & \\
\hline Cor da Pele & & & & $0,59^{* *}$ \\
\hline Preta & $7(58,3)$ & $4(33,3)$ & $11(45,8)$ & \\
\hline Parda & $4(33,3)$ & $7(58,3)$ & $11(45,8)$ & \\
\hline Branca & ---- & $1(8,3)$ & $1(4,2)$ & \\
\hline Amarela & $1(8,3)$ & --- & $1(4,2)$ & \\
\hline Consumo de Cigarro & & & & $1,0^{* *}$ \\
\hline Nunca & $9(75)$ & $8(66,7)$ & $17(71)$ & \\
\hline Ex-Fumante & $3(25)$ & $4(33,3)$ & $7(29)$ & \\
\hline Consumo de Álccol & & & & $0,11^{* *}$ \\
\hline Não & $5(41,7)$ & $9(75)$ & $14(58)$ & \\
\hline Uso de medicação anti-hipertensiva & & & & $0,59^{* *}$ \\
\hline Sim & $9(75)$ & $11(91,7)$ & $20(83)$ & \\
\hline \multicolumn{5}{|l|}{ Tipo de Medicação } \\
\hline Diurético Tiazídico (n=7) & $2(16,7)$ & $5(41,7)$ & $7(29)$ & $0,37^{* *}$ \\
\hline Beta-bloqueador $(n=10)$ & $3(25)$ & $7(58,3)$ & $10(42)$ & $0,21^{\text {** }}$ \\
\hline Inibidor da ECA $(n=1)^{*}$ & $1(8,3)$ & ---- & $1(4,2)$ & $1,0^{* *}$ \\
\hline $\operatorname{BRA}^{\ddagger}(n=15)$ & $8(66,7)$ & $6(50)$ & $14(58)$ & $0,68^{* *}$ \\
\hline Bloqueador dos Canais de $\mathrm{Ca}(\mathrm{n}=3)$ & $3(25)$ & $1(8,3)$ & $4(17)$ & $0,59^{* *}$ \\
\hline \multicolumn{5}{|l|}{ Classificação da PA } \\
\hline Controlada & $7(58,3)$ & $10(83,3)$ & $17(71)$ & $0,37^{\star *}$ \\
\hline
\end{tabular}

*Inibidor da ECA: Inibidor da Enzima Conversora de Angiotensina; ${ }^{\ddagger}$ BRA: Bloqueador do Receptor de Angiotensina II; ${ }^{\$}$ Teste T de Student; ${ }^{* *}$ Teste Exato de Fisher. 
Para variáveis pressóricas da MAPA, observou-se média de $154 \pm 19 \mathrm{mmHg}, 134 \pm 19 \mathrm{mmHg}$ e $125 \pm 16 \mathrm{mmHg}$ para pico durante vigília, sono e PAS média, respectivamente. Além disso, observou-se média de $105 \pm 11 \mathrm{mmHg}$, $91 \pm 13 \mathrm{mmHg}$ e $82 \pm 10 \mathrm{mmHg}$ para pico durante vigília, sono e PAD média. Considerando medidas de consultório, a média foi de $152 \pm 21 \mathrm{mmHg}, 92 \pm 16 \mathrm{mmHg}$ e $77 \pm 12 \mathrm{bpm}$ para PAS, PAD e FC, respectivamente. A maioria dos participantes não apresentou descenso pressórico durante o sono, sendo 58,3\%, no grupo controle e 66,7\% no grupo intervenção. Os valores iniciais das variáveis pressóricas não apresentaram diferenças entre os grupos (Tabela 2).

Tabela 2. Características basais da amostra de acordo com as variáveis de PA (MAPA). 2020. ( $n=24)$

\begin{tabular}{|c|c|c|c|c|}
\hline Variáveis & $\begin{array}{l}\text { Controle } \\
(n=12)\end{array}$ & $\begin{array}{l}\text { Intervenção } \\
(n=12)\end{array}$ & $\begin{array}{l}\text { Total } \\
(n=24)\end{array}$ & p-valor \\
\hline Pressão Arterial Sistólica (PAS) (mmHg) & $X \pm S D$ & $X \pm S D$ & $\mathrm{X} \pm \mathrm{SD}$ & \\
\hline Pico durante vigília & $156,6 \pm 15,7$ & $152,3 \pm 22,8$ & $154 \pm 19$ & 0,59 \\
\hline Pico durante sono & $132,8 \pm 12,6$ & $135,9 \pm 24,4$ & $134 \pm 19$ & 0,71 \\
\hline Média & $126,8 \pm 13,5$ & $122,8 \pm 19,3$ & $125 \pm 16$ & 0,56 \\
\hline \multicolumn{5}{|l|}{ Pressão Arterial Diastólica (PAD) (mmHg) } \\
\hline Pico durante vigília & $106,8 \pm 12,3$ & $103,2 \pm 8,5$ & $105 \pm 11$ & 0,41 \\
\hline Pico durante sono & $91,4 \pm 14,4$ & $90,8 \pm 12,3$ & $91 \pm 13$ & 0,92 \\
\hline Média & $85,1 \pm 11,7$ & $79,6 \pm 8,6$ & $82 \pm 10$ & 0,20 \\
\hline \multicolumn{5}{|l|}{ Ausência de Descenso Pressórico $(n=15)$} \\
\hline $\operatorname{sim}$ & $7(58,3 \%)$ & $8(66,7 \%)$ & $15(62,5)$ & $0,50^{+}$ \\
\hline
\end{tabular}

*Student $\mathrm{T}$ test; ${ }^{+}$Fisher Exact test.

A PRP não apresentou efeito imediato na queda da PA entre os tempos estudados para Gl ou GC (p>0,05). Na comparação intragrupo do GC, nenhuma variável pressórica alcançou diferença significante, mostrando que houve pouca alteração das variáveis durante as seis semanas de intervenção. Na comparação do Gl, a variável pico da PAS durante o sono apresentou diferença entre os níveis basais e após a intervenção, sendo a média de $135,8 \pm 24,4 \mathrm{mmHg}$, no período basal e de $129,1 \pm 25,2 \mathrm{mmHg}$, após a PRP $(p<0,05)$.

Na comparação intergrupos dos deltas, observou-se que o delta do pico da PAS durante vigília foi de $+9,3 \mathrm{mmHg}$ para GC e de $-7,5 \mathrm{mmHg}$ para GI $(p<0,05)$, com diferença de $16,8 \mathrm{mmHg}$ entre os grupos e TDE de 1,10 . $O$ delta do pico da PAS durante sono foi de $+2,3 \mathrm{mmHg}$ para GC e de $-6,8 \mathrm{mmHg}$ para $\mathrm{Gl}(p<0,05)$, com diferença entre os grupos de $9,1 \mathrm{mmHg}$ e TDE de 0,3 e o delta do pico da PAD durante vigília foi de $+3,2 \mathrm{mmHg}$ no GC e de $-4,7 \mathrm{mmHg}$ no $\mathrm{Gl}(\mathrm{p}<0,05)$, com diferença de 7,9mmHg e TDE de 1,12 (Tabela 3).

Tabela 3. Comparação Intergrupo dos deltas de PA (MAPA). 2020. ( $n=24)$

\begin{tabular}{llll}
\hline Variáveis & $\begin{array}{l}\text { Controle } \\
(\mathrm{n}=12)\end{array}$ & $\begin{array}{l}\text { Intervenção } \\
(\mathrm{n}=12)\end{array}$ & p-valor $^{*}$ \\
\hline Pressão Arterial Sistólica (PAS) $(\mathrm{mmHg})$ & Dif(Pós-Pre) & Dif(Pós-Pre) & 0,04 \\
$\quad \begin{array}{l}\Delta \text { Pico durante vigília } \\
\quad \text { Pico durante sono }\end{array}$ & $+9,3$ & $-7,5$ & 0,03 \\
$\quad$ Média & $+2,3$ & $-6,8$ & 0,20 \\
Pressão Arterial Diastólica (PAD) $(\mathrm{mmHg})$ & $+3,0$ & $-3,8$ & 0,04 \\
$\quad \begin{array}{l}\Delta \text { Pico durante vigília } \\
\text { P Pico durante sono }\end{array}$ & $+3,2$ & & 0,52 \\
$\quad$ Média & $-1,3$ & $-4,7$ & 0,82 \\
\hline
\end{tabular}

*Student T test. 
Para análise de postura, foram inscritos 30 participantes, que mantiveram as mesmas características de frequência. Para os valores basais dos parâmetros de postura, os participantes apresentaram deslocamento do tronco para posterior, deslocamento do corpo para anterior, ângulos do quadril e do joelho aumentados e do tornozelo diminuído, sem diferença entre os grupos $(p>0,05)$ (Tabela 4). Tanto na comparação intragrupos, como intergrupos, os parâmetros basais se mantiveram, para GC e Gl, sem diferença estatística. Após recategorização, observou-se melhora semelhante entre os dois grupos, sem diferença estatística $(p>0,05)$.

Tabela 4. Características basais de acordo com os Ângulos de Postura (SAPO) $(n=30)$

\begin{tabular}{|c|c|c|c|}
\hline Variáveis & $\begin{array}{c}\text { Controle } \\
(n=15)\end{array}$ & $\begin{array}{l}\text { Intervenção } \\
(n=15)\end{array}$ & p-valor ${ }^{+}$ \\
\hline & Med[IQ25-75] $]^{*}$ & Med[IQ25-75 & \multirow{6}{*}{$\begin{array}{c}0,78 \\
1,0 \\
0,94 \\
1,0 \\
0,94\end{array}$} \\
\hline Alinhamento Vertical do Tronco (AVT) & $-1,7[-3,4-2,4]$ & $-1,5[-3,4-2,1]$ & \\
\hline Alinhamento Vertical do Corpo (AVCo) & $2,5[1,4-3,0]$ & $2,1[1,1-3,2]$ & \\
\hline Ângulo do Quadril (AQ) & $-6,5[-12,4--0,4]$ & $-5,5[-11,2--1,6]$ & \\
\hline Ângulo do Joelho (AJ) & $-0,7[-3,6-2,0]$ & $-1,7[-3,8-4,2]$ & \\
\hline Ângulo do Tornozelo (AT) & $85,4[84,3-87,5]$ & $85,9[83,3-86,9]$ & \\
\hline
\end{tabular}

*Mediana[Intervalo Interquartil]; ${ }^{+}$Mann-Whitney

Para o Gl, deslocamento do corpo anterior apresentou moderada correlação positiva para PAS ( $r=0,57 ; p=0,03)$, $33 \%$ da PAS explicada pelo deslocamento anterior do corpo. Ângulo do joelho com correlação moderada positiva para PAD ( $r=0,68 ; p<0,01)$ e Ângulo de tornozelo com correlação moderada negativa para PAD $(r=-0,75 ; p<0,01)$, com $46 \%$ e 55\% da PAD explicada pelos ângulos, respectivamente. (Figura 2). Não foram encontradas correlações entre parâmetros específicos de postura e PA considerando os valores iniciais nos dois grupos e os valores finais do GC.

Figura 3. Comparação intergrupo dos deltas de Pico durante Vigília e Sono das variáveis pressóricas (MAPA).
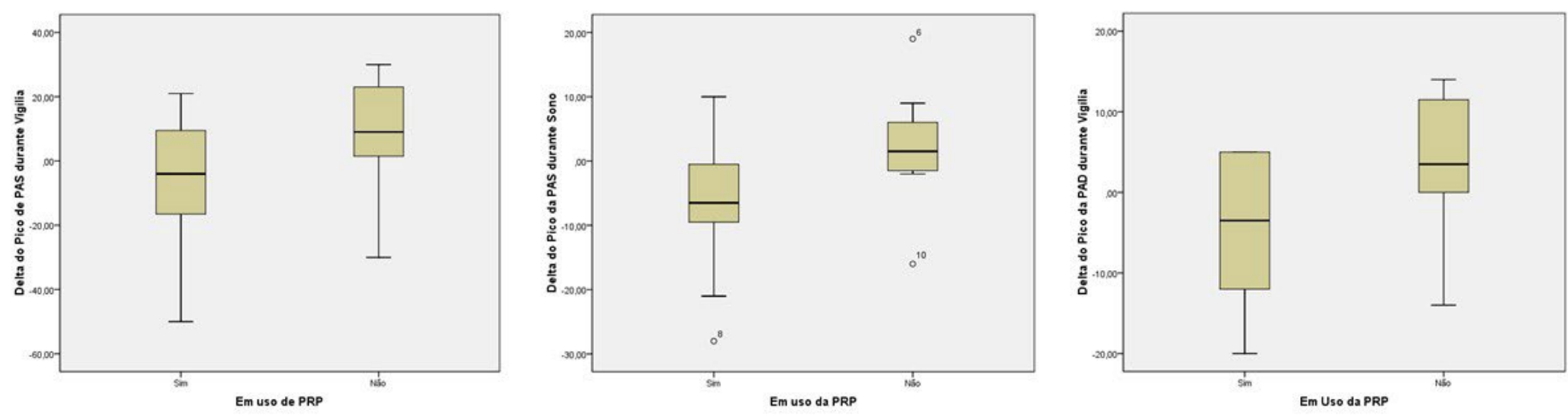

\section{Discussão}

Esta pesquisa teve como objetivo verificar o efeito da PRP na queda da PA em indivíduos hipertensos e após seis semanas de uso da PRP, não foi observado qualquer efeito sobre a média da PA, observou-se grande efeito na redução do pico da PAS durante a vigília, efeito moderado na redução do pico da PAS durante o sono e grande efeito na redução do pico da PAD durante a vigília. A corrente elétrica provocada pelo relevo das palmilhas tem ação tanto nos músculos adjacentes (sistema somatosensorial profundo) ${ }^{13}$ e nas propriedades plasmáticas dos fluidos ${ }^{25,26}$.

É possível que a posição do dispositivo no pé tenha causado reflexo miotático direto no músculo que recebe o estímulo ${ }^{13}$. Estudos que correlacionaram a Atividade Nervosa Simpática Muscular (ANSM) e hipertensão arterial demonstraram, em sua maioria, um aumento do ANSM em repouso nos músculos, atividade simpática sistêmica, com liberação de noradrenalina e glutamato circulantes, ativação do $\beta$-adrenoceptores, que aumentará o tônus vascular e a resistência vascular periférica (RVP), 4,6,27,28, 
Neste estudo, foi possível observar que houve diminuição dos picos pressóricos ${ }^{21}$. Os picos de pressão estão associados a descargas adrenérgicas, secundárias à estimulação simpática. Uma vez que os picos pressóricos diminuíram para a PAS em períodos de vigília e de sono e para a PAD durante vigília, em torno de $17 \mathrm{mmHg}, 9 \mathrm{mmHg}$ e $8 \mathrm{mmHg}$, respectivamente, com magnitude de efeito moderada ou grande, podese sugerir que houve inibição do estímulo simpático.

É importante ressaltar os tamanhos dos efeitos produzidos pelo PRP. Em uma revisão sistemática e metanálise de 13 estudos publicados, combinando intervenções dietéticas e atividade física de média a alta intensidade, com a maioria dos participantes tomando drogas anti-hipertensivas, a média basal da PAS/PAD foi de 127-162/71-96mmHg para indivíduos usando medicação e 144/87mmHg no grupo sem uso de drogas e duração da intervenção entre 12-24 meses mostrou uma redução da PAS e da PAD por um período de $<12$ meses de 2. $29(3,82-0,76) \mathrm{mmHg}$ e de $1,22(2,53$ a 0,08$) \mathrm{mmHg}$, respectivamente ${ }^{22}$. O presente estudo mostrou uma queda máxima do pico da PAS de pelo menos $9 \mathrm{mmHg}$ e da PAD em torno de $8 \mathrm{mmHg}$.

Outra hipótese para entender os resultados é a possibilidade da corrente eletromagnética produzida pela PRP estar envolvida na regulação da atividade simpática sistêmica. O propósito do ressonador é provocar a despolarização dos neurônios na região próxima à localização do dispositivo, gerando potenciais de ação, os quais serão levados ao cérebro ${ }^{29}$. Embora esta teoria pareça ser viável, não há certeza de seu funcionamento para a PRP. A palmilha tem como característica promover corrente de baixa freqüência com fluxo de elétrons constante ${ }^{12}$.

Estudos que aplicaram estimulação transcutânea do nervo vago (tVNS), com eletrodos colocados em tragus, mostraram que a freqüência cardíaca em repouso diminuiu e a sensibilidade barorreflexa cardíaca aumentou, regulando a modulação autonômica ${ }^{30}$. Além disso, tVNS pode alterar controle autonômico cardiovascular em humanos saudáveis, por meio da mudança no equilíbrio autonômico cardíaco, gerando dominância parassimpática/vagal (tVNS diminuiu significativamente a relação LF/HF), e diminuição do ANSM $^{31}$. A estimulação elétrica nervosa transcutânea (TENS) melhora o fluxo sanguíneo ${ }^{32}$. TENS de baixa freqüência diminuiu atividade no sistema nervoso simpático e aumentou no parassimpático, quando aplicada na região paravertebral ganglionar de pacientes hipertensos ${ }^{33}$.

Considerando a melhora da postura, após seis semanas de uso da PRP, notou-se pouca mudança na postura, com manutenção dos padrões posturais iniciais, para ambos os grupos. Este estudo observou que o deslocamento corporal anterior revelou uma associação moderada e positiva à PAS (quanto maior a projeção do corpo para frente, maior o valor da PA).

Em estudo anterior, desalinhamentos posturais mostraram associação com elementos MAPA: o deslocamento anterior do tronco apresentou menor variação vigília/sono para PAS (14,7\%Vs25,3\%, $p=0,01)$, o quadril flexionado apresentou maior carga pressórica $(29,4 \% V s 18,3 \% p=0,02)$ para PAD(19). Diferenças nas alterações dos ângulos posturais podem levar a diferentes associações com as variáveis pressóricas, mas a suposição de que os desalinhamentos posturais podem modificar a regulação da PA torna-se mais consistente.

A diminuição do deslocamento anterior do corpo e consequente melhora da distribuição do centro de massa pode impactar a intensidade da contração muscular de toda a cadeia posterior, que se contrai para que as pessoas possam se manter contra a gravidade. A diminuição da contração muscular pode diminuir o ANSM, com potencial diminuição da atividade simpática sistêmica e consequente diminuição da PA ${ }^{4,6}$.

Os ângulos do joelho e do tornozelo são proporcionais em posição de pé: quanto menor o ângulo do joelho (mais flexão), menor o ângulo do tornozelo (mais dorsiflexão). A diminuição dos ângulos causou um aumento da PAD. Os ângulos acima mencionados geram alongamento excêntrico da musculatura posterior da perna, principalmente gastrocnêmio e sola, alterando o estado de tensão muscular, com compressões mecânicas sustentadas dos vasos e estímulo mecanorreceptor ${ }^{34}$. Este estado constante de tensão da musculatura posterior da perna causa a perda do mecanismo da bomba de panturrilha. Esta bomba tem um papel importante no retorno venoso e na interação dos sistemas cardio-postural-musculo-esquelético ${ }^{35}$. 
A PRP não afetou a correção da postura, mas melhorou o deslocamento anterior do corpo, o que favoreceu uma diminuição da PAS. A manutenção da flexão do joelho e da dorsiflexão do tornozelo teve impacto no aumento da PAD. É importante ressaltar que o dispositivo não deve ser a única correção a ser realizada na palmilha para adequação postural completa: calços são normalmente usados para corrigir dismetrias dos membros inferiores, pés planos e valgos ${ }^{13}$, o que poderia ter impactado na melhora da postura. Entretanto, o principal objetivo deste estudo foi verificar o efeito do dispositivo, isoladamente, na postura de indivíduos hipertensos e verificar se a mudança na postura poderia afetar a PA.

Como limitações este estudo não atingiu o número estimado pelo cálculo do tamanho da amostra e a necessidade de considerar como critério de exclusão os participantes que não realizaram MAPA ao final de seis semanas de intervenção. Um grande número de pacientes hipertensos apresentou obesidade, síndrome metabólica e diabetes mellitus, todos critérios de exclusão do estudo. Essas condições clínicas afetam o sistema neuroendócrino e são consideradas, para esses estudos, modificadores de resultados, pois interferem nos mecanismos neurofisiológicos que potencialmente explicam os resultados da intervenção.

Considerando a importância da MAPA para o desfecho principal e por se tratar de um estudo exploratório, os dados do exame foram necessários para entender a real ação da palmilha sobre esta população. Entretanto, é importante notar que este estudo utilizou um desenho adequado de ensaio clínico randomizado para testar o efeito da PRP.

A originalidade deste estudo foi propor uma forma inovadora de produzir corrente eletromagnética através da combinação de metais em uma palmiIha, com ação focada no sistema cardiovascular. Considerando os resultados positivos, é importante incentivar o uso desta ferramenta em uma população maior, com menor controle das variáveis intervenientes, para identificar o comportamento da PRP diante destas condições.

A partir dos resultados, é possível considerar que a PRP pode ser um tratamento complementar para indivíduos com hipertensão arterial para reduzir os picos de pressão da PAS e da PAD durante o período de vigília.

\section{Contribuições dos autores}

Góes ALB participou da concepção, delineamento, busca e análise estatística dos dados da pesquisa, interpretação dos resultados e redação do artigo científico. Barbosa ASM, Araújo BGV, Barbosa CC, Barbalho GMOG, Lago VC, Souza LAP participaram da coleta e interpretação dos dados da pesquisa. Ladeia AMT participou da concepção, delineamento, busca e análise estatística dos dados da pesquisa, interpretação dos resultados, redação e edição do artigo científico.

\section{Conflitos de interesses}

Nenhum conflito financeiro, legal ou político envolvendo terceiros (governo, empresas e fundações privadas, etc.) foi declarado para nenhum aspecto do trabalho submetido (incluindo, mas não se limitando a subvenções e financiamentos, participação em conselho consultivo, desenho de estudo, preparação de manuscrito, análise estatística, etc.).

\section{Referências}

\begin{abstract}
1. Mozaffarian D, Benjamin EJ, Go AS, Arnett DK, Blaha MJ, Cushman M et al. Heart Disease and Stroke Statistics-2015 Update: A Report from the American Heart Association. Circulation. 2015;131:e29-e322. doi: $10.1161 /$ CIR.0000000000000152
\end{abstract}

2. Malachias MVB, Gomes MAM, Nobre F, Alessi A, Feitosa AD, Coelho EB. 7a Diretriz Brasileira de Hipertensão Arterial. Arq Bras Cardiol. 2016;107(3):1-83. doi: 10.5935/abc.20160152

3. Lobo LAC, Canuto R, Dias-da-Costa JS, Pattussi MP. Tendência temporal da prevalência de hipertensão arterial sistêmica no Brasil. Cad Saude Publica. 2017;33(6): e00035316. doi: 10.1590/0102-311×00035316

4. Bruno RM, Ghiadoni L, Seravalle G, Dell'Oro R, Taddei S, Grassi
G. Sympathetic regulation of vascular function in health and
disease. Front Physiol. 2012;3:284. doi: $10.3389 /$ fphys.2012.00284

5. Fisher JP, Fadel PJ. Therapeutic strategies for targeting excessive central sympathetic activation in human hypertension. Exp Physiol. 2012;95(5):572-80. doi: 10.1113/expphysiol.2009.047332

6. Tsioufis C, Kordalis A, Flessas D, Anastasopoulos I, Tsiachris D, Papademetriou $V$ et al. Pathophysiology of resistant hypertension: the role of sympathetic nervous system. Int J Hypertens. 2011;2011: 642416. doi: $10.4061 / 2011 / 642416$

7. Wallin BG, Charkoudian N. Sympathetic neural control of integrated cardiovascular function: insights from measurement of human sympathetic nerve activity. Muscle Nerve. 2007;36(5):595614. doi: $10.1002 /$ mus.20831 
8. Pinheiro CHJ, Medeiros RAR, Pinheiro DGM, Marinho MJF. Modificação do padrão respiratório melhora o controle cardiovascular na hipertensão essencial. Arq Bras Cardiol. 2007;88(6):651-9. doi: 10.1590/50066-782X2007000600005

9. Irigoyen MC, Consolim-colombo FM, Krieger EM. Controle cardiovascular: regulação reflexa e papel do sistema nervoso simpático. Rev Bras Hipertens. 2001;8(1):55-62.

10. Mochizuki L, Amadio AC. As informações sensoriais para o controle postural. Fisioter Mov. 2006;19(2):11-8.

11. Horak FB. Postural orientation and equilibrium: What do we need to know about neural control of balance to prevent falls? Age Ageing. 2006;35(Suppl.2):7-11. doi: 10.1093/ageing/afl077

12. Bricot B. United States Patent (19) SYS332: Method and Pedal apparatus for spinal disorders. 1992. p. 12.

13. Bricot B. Posturologia Clinica. 1st ed. São Paulo: CIES Brasil; 2010.

14. Kavounoudias A, Roll R, Roll JP. The plantar sole is a "dynamometric map" for human balance control. Neuroreport. 1998;9(14):3247-52. doi: 10.1097/00001756-199810050-00021

15. Kavounoudias A, Roll R, Roll JP. Foot sole and ankle muscle inputs contribute jointly to human erect posture regulation. J Physiol. 2001;532(3):869-78. doi: 10.1111/j.14697793.2001.0869e.x

16. Ribot-Ciscar E, Roll JP. Ago-antagonist muscle spindle inputs contribute together to joint movement coding in man. Brain Res. 1998;791(1-2):167-76. doi: 10.1016/S0006-8993(98)00092-4

17. Roll JP, Bergenheim M, Ribot-Ciscar E. Proprioceptive population coding of two-dimensional limb movements in humans: II. Muscle-spindle feedback during "drawing-like" movements. Exp Brain Res. 2000;134(3):311-21. doi: 10.1007/ $\underline{\mathrm{s} 002210000472}$

18. Roll R, Kavounoudias A, Roll J-P. Cutaneous afferents from human plantar sole contribute to body posture awareness. Neuroreport. 2002;13(15):1957-61. doi: 10.1097/00001756200210280-00025

19. Goes ALB, Jesus DM, Silva TB, Lago VC, Souza LAP, Ladeia AMT. Influence of postural misalignment on blood pressure in hypertensive individuals: an exploratory cross sectional study. J Phys Res. 2018;8(2):111-20. doi: 10.17267/2238-2704rpf.v8i2.1955

20. Schulz KF, Altman DG, Moher D. CONSORT 2010 statement: updated guidelines for reporting parallel group randomised trials. BMJ. 2010;340:332. doi: 10.1136/bmj.c332
21. Sociedade Brasileira de Cardiologia. V Diretrizes de Monitorização Ambulatorial da Pressão Arterial (MAPA) e III Diretrizes de Monitorização Residencial da Pressão Arterial (MRPA). Arq Bras Cardiol. 2011;97(3):0-40. doi: 10.1590/S0066782X2011001800001

22. Lin JS, O'Connor EA, Evans CV, Senger CA, Rowland MG, Groom HC. Behavioral Counseling to Promote a Healthy Lifestyle for Cardiovascular Disease Prevention in Persons With Cardiovascular Risk Factors: An Updated Systematic Evidence Review for the U.S. Preventive Services Task Force. Agency Healthc Res Qual. 2014;(113).

23. Espirito-Santo H, Daniel F. Calcular e apresentar tamanhos do efeito em trabalhos científicos ( 1 ): As limitações do $p<0,05$ na análise de diferenças de médias de dois grupos. Rev Port Investig Comport e Soc. 2015;1(1):3-16.

24. Daniel T, Kleinjan K, Gillenwaters A, Mitchell W. Tables and Calculators - RStats Institute - Missouri State University [Internet]. [acesso em 2018 mar. 13]. Disponível em: https://www. missouristate.edu/rstats/Tables-and-Calculators.htm

25. Burnstock G. Purinergic signalling: From discovery to current developments. Exp Physiol. 2014;99(1):16-34. doi: 10.1113/ expphysiol.2013.071951

26. Luther JA, Birren SJ. Neurotrophins and target interactions in the development and regulation of sympathetic neuron electrical and synaptic properties. Auton Neurosci. 2009;151(1):46-60. doi: 10.1016/j.autneu.2009.08.009

27. Hammam E, Macefield VG. Vestibular modulation of sympathetic nerve activity to muscle and skin in humans. Front Neurol. 2017;8(334):1-14. doi: 10.3389/fneur.2017.00334

28. Leblanc $C$, Tabrizchi R. Role of $\beta 2$-and $\beta 3$-adrenoceptors in arterial stiffness in a state of hypertension. Eur J Pharmacol. 2018;819:136-43. doi: 10.1016/j.ejphar.2017.11.050

29. Nitsche MA, Fricke K, Henschke U, Schlitterlau A, Liebetanz D, Lang $\mathrm{N}$ et al. Pharmacological modulation of cortical excitability shifts induced by transcranial direct current stimulation in humans. J Physiol. 2003;533(1):293-301. doi: 10.1113/ jphysiol.2003.049916

30. Antonino D, Teixeira AL, Maia-Lopes PM, Souza MC, SabinoCarvalho JL, Murray AR et al. Non-invasive vagus nerve stimulation acutely improves spontaneous cardiac baroreflex sensitivity in healthy young men: A randomized placebo-controlled trial. Brain Stimul. 2017;10(5)875-81. doi: 10.1016/j.brs.2017.05.006

31. Clancy JA, Mary DA, Witte KK, Greenwood JP, Deuchars SA, Deuchars J. Non-invasive Vagus Nerve Stimulation in Healthy Humans reduces Sympathetic Nerve Activity. Brain Stimul. 2014;7(6):871-7. doi: 10.1016/j.brs.2014.07.031 
32. Cipriano Jr G, Neder JA, Umpierre D, Arena R, Vieira PJC, Chiappa AMG et al. Sympathetic ganglion transcutaneous electrical nerve stimulation after coronary artery bypass graft surgery improves femoral blood flow and exercise tolerance. J Appl Physiol. 2014;117(6):633-8. doi: $10.1152 /$ japplphysiol.00993.2013

33. Sartori SA, Stein C, Coronel CC, Macagnan FE, Plentz RDM. Effects of Transcutaneous Electrical Nerve Stimulation in Autonomic Nervous System of Hypertensive Patients: A Randomized Controlled Trial. Curr Hypertens Rev. 2018;14(1):6671. doi: $10.2174 / 1573402114666180416155528$
34. Proske U, Gandevia SC. The Proprioceptive Senses: Their Roles in Signaling Body Shape, Body Position and Movement, and Muscle Force. Physiol Rev. 2012;92(4):1651-97. doi: 10.1152/ physrev.00048.2011

35. Verma AK, Garg A, Xu D, Bruner M, Fazel-Rezai R, Blaber AP et al. Skeletal Muscle Pump Drives Control of Cardiovascular and Postural Systems. Sci Rep. 2017;7(45301):1-8. doi: 10.1038/ srep45301 